\title{
Immunotherapy beyond progression in patients with advanced non-small cell lung cancer
}

\author{
Xiangwei Ge ${ }^{1,2 \#}$, Zhibo Zhang ${ }^{2,3 \#}$, Sujie Zhang ${ }^{4 \#}$, Fang Yuan ${ }^{4}$, Fan Zhang ${ }^{4}$, Xiang Yan ${ }^{4}$, Xiao Han ${ }^{4}$, \\ Junxun $\mathrm{Ma}^{4}$, Lijie Wang ${ }^{4}$, Haitao Tao ${ }^{4}$, Xiaoyan $\mathrm{Li}^{4}$, Xiaoyu Zhi ${ }^{1,2}$, Zhiyue Huang ${ }^{5}$, Paul Hofman ${ }^{6}$, \\ Arsela Prelaj ${ }^{7,8}$, Giuseppe Luigi Banna ${ }^{9}$, Luciano Mutti ${ }^{10}$, Yi $\mathrm{Hu}^{4}$, Jinliang Wang ${ }^{2,4}$
}

${ }^{1}$ Medical School of Chinese PLA, Beijing, China; ${ }^{2}$ Department of Oncology, the Second Medical Center, Chinese PLA General Hospital, Beijing, China; ${ }^{3}$ The 78th Group Army Hospital of Chinese PLA, Mudanjiang, China; ${ }^{4}$ Department of Oncology, Chinese PLA General Hospital, Beijing, China; ${ }^{5}$ BeiGene (Shanghai) Co., Ltd., Shanghai, China; ${ }^{6}$ Laboratory of Clinical and Experimental Pathology, Pasteur Hospital, Nice, France; ${ }^{7}$ Medical Oncology Department, Fondazione IRCCS Istituto Nazionale Tumori, Milan, Italy; ${ }^{8}$ Department of Electronics, Information, and Bioengineering, Polytechnic University of Milan, Milan, Italy; ${ }^{9}$ Department of Oncology, Portsmouth Hospitals University NHS Trust, Portsmouth, UK; ${ }^{10}$ Center for Biotechnology, Sbarro Institute for Cancer Research and Molecular Medicine, College of Science and Technology, Temple University, Philadelphia, PA, USA

Contributions: (I) Conception and design: X Ge, Z Zhang, Y Hu, J Wang; (II) Administrative support: J Wang, Y Hu; (III) Provision of study materials or patients: All authors; (IV) Collection and assembly of data: X Ge, Z Zhang; (V) Data analysis and interpretation: Z Huang, X Ge, Z Zhang; (VI) Manuscript writing: All authors; (VII) Final approval of manuscript: All authors.

\#These authors contributed equally to this work.

Correspondence to: Dr. Jinliang Wang, MD. Department of Oncology, the Second Medical Center, Chinese PLA General Hospital, Beijing, China.

Email: wangjinliang301@163.com; Dr. Yi Hu, MD. Department of Oncology, Chinese PLA General Hospital, Beijing, China.

Email: huyi301zlxb@sina.com.

Background: Immune checkpoint inhibitors (ICIs) represent a great breakthrough in the treatment of advanced non-small cell lung cancer (aNSCLC). However, whether immunotherapy beyond progression (IBP) is effective for aNSCLC has yet to be established. Therefore, a retrospective clinical study was conducted to investigate the efficacy of IBP in patients with aNSCLC under real-world conditions.

Methods: A total of 125 Chinese patients with aNSCLC who experienced progressive disease (PD) after receiving monotherapy or combination therapy (combined with chemotherapy or/and antiangiogenic therapy) with programmed cell death-1 (PD-1)/programmed cell death ligand-1 (PD-L1) inhibitors between January 2015 and March 2019 were enrolled. Patients who were treated with ICIs for more than 6 weeks after PD were defined as IBP $(n=39)$, while those who received ICI treatment for less than 6 weeks or discontinued it due to PD were defined as non-IBP $(n=86)$. Patient clinical characteristics were evaluated. An optimization-based method was applied to balance the clinical baseline characteristics between the two groups.

Results: In total population, the IBP group had longer overall survival (median OS, 26.6 vs. 9.5 months; HR, 0.40; 95\% CI: 0.23-0.69; $\mathrm{P}<0.001$ ) and progression-free survival (median PFS, 8.9 vs. 4.1 months; HR, 0.41; 95\% CI: 0.26-0.65; $\mathrm{P}<0.001)$, compared with the non-IBP group. Despite no significant difference in objective response rate (ORR, $15.4 \%$ vs. $11.6 \%, \mathrm{P}=0.560)$, disease control rate (DCR) was significantly higher in the IBP group $(89.7 \%$ vs. $61.6 \%, \mathrm{P}<0.001)$. After balancing baseline covariates, the IBP group also had longer OS (median: 26.6 vs. 10.7 months; HR, 0.40; 95\% CI: 0.19-0.84; P=0.015) and PFS (median: 9.7 vs. 4.3 months; HR, 0.28; 95\% CI: $0.15-0.51$; $<<0.001$ ), with a benefit in either of patients previously treated with ICI monotherapy or in combination therapy and with non-response to the previously ICI.

Conclusions: IBP is associated with longer OS and PFS in patients with aNSCLC. Our findings may suggest new therapeutic options for patients with aNSCLC who experienced disease progression after initial immunotherapy. 


\begin{abstract}
Keywords: Immunotherapy beyond progression; non-small cell lung cancer (NSCLC); immune checkpoint inhibitors (ICIs); prognosis
\end{abstract}

Submitted Sep 14, 2020. Accepted for publication Dec 18, 2020.

doi: $10.21037 /$ tlcr-20-1252

View this article at: http://dx.doi.org/10.21037/tlcr-20-1252

\section{Introduction}

Lung cancer is one of the most common malignancies in the world. In 2018, lung cancer was responsible for approximately 1.8 million deaths globally, with 2.1 million new cases diagnosed (1). There are two main subtypes of lung cancer: non-small cell lung cancer (NSCLC) and small cell lung cancer. NSCLC is the most common histological type, accounting for more than $85 \%$ of lung cancer, and has a 5 -year survival rate of less than $16 \%$ (2). Therefore, there is an urgent need for improving the survival of patients with advanced non-small cell lung cancer (aNSCLC).

Immune checkpoint inhibitors (ICIs), including antiprogrammed cell death-1 (PD-1) and anti-programmed cell death ligand-1 (PD-L1) antibodies, have made a major impact on the treatment landscape for patients with aNSCLC in recent years (3-6). In a series of clinical trials, some ICIs were confirmed to achieve a better clinical response than standard chemotherapy in patients with aNSCLC (7-9). However, only a limited number of patients obtain sustained benefit from ICI therapy (10). For patients with disease progression to treatment with ICIs, the continuation of immunotherapy with subsequent treatment regimens is still investigational.

Immunotherapy beyond progression (IBP) has been reported as possibly effective for advanced melanoma and renal cell carcinoma patients (11-13). In the subgroup analysis of Checkmate 025 study, for instance, the tumor burden was reduced by $30 \%$ or more in $13 \%(20 / 153)$ of patients with advanced renal cell carcinoma in the IBP group (14). Moreover, in a retrospective melanoma study (15), the median overall survival (mOS) of patients who received IBP with anti-PD-1 antibody was significantly longer than that of patients in the non-IBP group. Conversely, research on IBP with ICIs in NSCLC is still in its infancy. In the long-term analysis of Keynote-010, 7 of 14 patients who received IBP with pembrolizumab achieved a partial response (PR) or stable disease (SD) as their best response, suggesting that IBP may hold potential for the treatment of patients with aNSCLC (16). While in a realworld European series of 60 aNSCLC patients with PD-L1 $\geq 50 \%$ progressed to first-line pembrolizumab and treated with salvage chemotherapy (42/60, $70 \%)$ or pembrolizumab and possible local ablative radiotherapies $(18 / 60,30 \%)$, the post-progression median survival between these two groups was not significantly different (6.9 vs. 8.1 months, $\mathrm{P}=0.08)(17)$. Therefore, further research on IBP is necessary.

In this context, we conducted a retrospective clinical study under real-world conditions to investigate whether IBP was effective for patients with aNSCLC and to further identify those subgroups of patients that could potentially benefit more from this treatment. We present the following article in accordance with the STROBE reporting checklist (available at http://dx.doi.org/10.21037/tlcr-20-1252).

\section{Methods}

\section{Study design and patients}

The study was conducted in accordance with the Declaration of Helsinki (as revised in 2013). The study was approved by the Ethics Committee of PLA General Hospital (S2018-141-01) and individual consent for this retrospective analysis was waived.

Between January 2015 and March 2019, patients with aNSCLC whose response was evaluated as progressive disease (PD) after receiving monotherapy or combination therapy (combined with chemotherapy or/and antiangiogenic therapy) with PD-1/PD-L1 checkpoint inhibitors at the Chinese PLA general hospital were screened. The inclusion criteria were: (I) NSCLC confirmed by pathological diagnosis; (II) stage IIIB or IV or recurrent disease; (III) at least one cycle of ICI treatment. Patients without disease reassessment to ICI treatment were excluded.

Patients' electronic medical records were reviewed, and 125 patients were eligible for this analysis. Patients who were treated with ICIs for more than 6 weeks after PD were defined as IBP, while those who received ICI treatment for less than 6 weeks or discontinued it due to the PD were defined as non-IBP.

The clinical data of the patients were collected, including 
age, gender, tumor histology, smoking history, Eastern Cooperative Oncology Group (ECOG) performance status, tumor stage, liver/brain metastases, previous lines of therapy, best response to the previous line, initial immunotherapy regimen (monotherapy/combination therapy). ICIs used included nivolumab, pembrolizumab, atezolizumab, and durvalumab. Tumor response, including complete response (CR), $\mathrm{PR}, \mathrm{SD}$ and $\mathrm{PD}$, were assessed by referring to the Response Evaluation Criteria in Solid Tumors (RECIST) criteria version 1.1 (18).

The primary study objective was overall survival (OS), defined as the time from the initiation of the postPD treatment to death from any cause. The secondary objectives were progression-free survival (PFS), objective response rate (ORR), and disease control rate (DCR). PFS was defined as the time from the initiation of the post-PD treatment to disease progression or death from any cause, whichever came first. The ORR was defined as the sum of CR and PR, while the DCR was the sum of CR, PR, and SD. The date of the last follow-up was May 1, 2020.

\section{Statistical analysis}

An optimization-based method was used to eliminate the standardized mean difference in an attempt to balance the distribution of covariates between the IBP and non-IBP groups (19). A weight was assigned to patients according to the following criteria: (I) the variance ratio was between 0.67 and 2.0; (II) the absolute value of the standardized mean difference was less than 0.15 . Kish's approximate formula was used to calculate the effective size for the weighted sample. Chi-square or Fisher's exact test was used to compare categorical variables. Continuous or ordinal variables were compared using the Student's $t$-test or Mann-Whitney U test. Kaplan-Meier curves were used to analyze OS and PFS, and the log-rank test was used to compare the survival curves. Cox proportional-hazards regression was performed to calculate the hazard ratios (HRs). P-values were calculated based on a two-sided assumption, and $\mathrm{P}<0.05$ was considered to be statistically significant. Statistical analyses were performed using $\mathrm{R}$ for the optimization-based method and survey for the weighted sample (R packages WeightIt version 0.5.1, https://cran. r-project.org/web/packages/WeightIt/index.html; survey version 3.36, https://cran.r-project.org/web/packages/ survey/index.html).

\section{Results}

\section{Patient clinical characteristics}

A total of 125 patients with aNSCLC who experienced PD after treatment with PD-1/PD-L1 inhibitors were enrolled in this study. There were 39 and 86 patients in the IBP group and the non-IBP group, respectively. Table 1 summarized the patients' baseline clinical characteristics. The median age for all participants was 59 (range, 33-82) years; $91(72.8 \%)$ patients were male and $34(27.2 \%)$ were female.

Table 1 Baseline characteristics of the patients

\begin{tabular}{lccc}
\hline \multirow{2}{*}{ Characteristics } & \multicolumn{3}{c}{ No. of patients (\%) } \\
\cline { 2 - 4 } & All patients $(\mathrm{n}=125)$ & IBP group $(\mathrm{n}=39)$ & Non-IBP group $(\mathrm{n}=86)$ \\
\hline Median age (range), years & $59(33-82)$ & $56(33-82)$ & $59(33-79)$ \\
$\quad$ & & \\
Male & $91(72.8)$ & $29(74.4)$ & $62(72.1)$ \\
Female & $34(27.2)$ & $10(25.6)$ & $24(27.9)$ \\
ECOG performance status & & & \\
$0-1$ & $105(84.0)$ & $36(92.3)$ & $69(80.2)$ \\
$\geq 2$ & $20(16.0)$ & $3(7.7)$ & $17(19.8)$ \\
Smoking history & & & $52(60.5)$ \\
Ever & $75(60.0)$ & $23(59.0)$ & $34(39.5)$ \\
Never & $50(40.0)$ & $16(41.0)$ & \\
\hline
\end{tabular}

Table 1 (continued) 
Table 1 (continued)

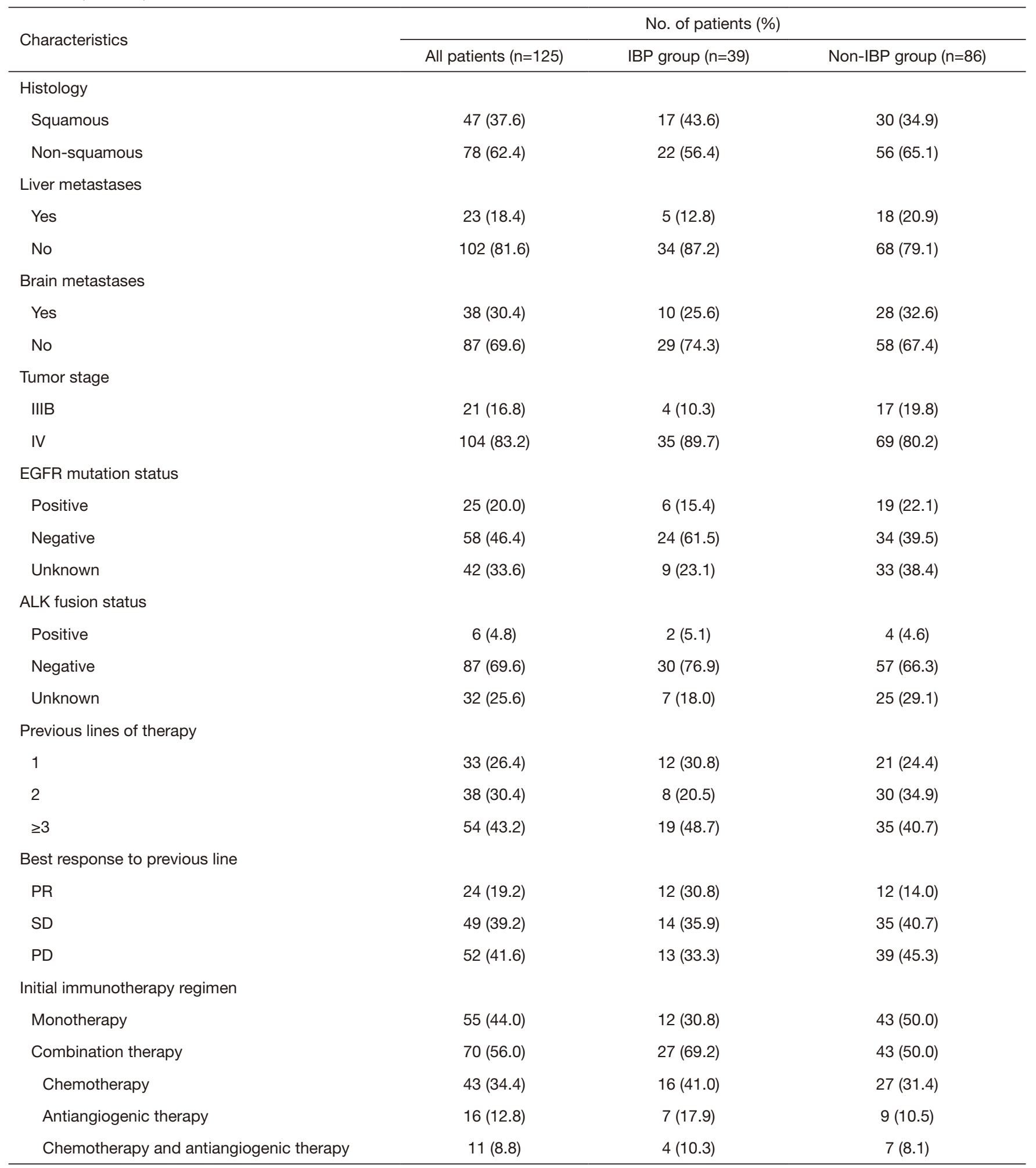

ECOG, Eastern Cooperative Oncology Group; IBP, immunotherapy beyond progression; PR, partial response; SD, stable disease; PD, progressive disease. 
Table 2 Tumor response in all patients

\begin{tabular}{lccc}
\hline Best response & IBP group & Non-IBP group & P value \\
\hline CR, n (\%) & $0(0)$ & $0(0)$ & \\
PR, n (\%) & $6(15.4)$ & $10(11.6)$ & \\
SD, n (\%) & $29(74.3)$ & $43(50.0)$ & \\
PD, n (\%) & $4(10.3)$ & $33(38.4)$ & \\
ORR, n (\%) & $6(15.4)$ & $10(11.6)$ & 0.560 \\
DCR, n (\%) & $35(89.7)$ & $53(61.6)$ & 0.001 \\
\hline
\end{tabular}

$\mathrm{CR}$, complete response; $\mathrm{PR}$, partial response; SD, stable disease; PD, progressive disease; ORR, objective response rate; $\mathrm{DCR}$, disease control rate; IBP, immunotherapy beyond progression.

$75(60.0 \%)$ patients had a smoking history. 47 (37.6\%) patients had squamous cell carcinoma and 78 (62.4\%) patients had non-squamous cell carcinoma. Liver and brain metastases were presented in 23 (18.4\%) and 38 (30.4\%) patients, respectively. 25 (20\%) patients had positive EGFR mutations status, and $6(4.8 \%)$ patients had positive ALK fusion status. Of the initial immunotherapy regimens, combination therapy was found to be more common than monotherapy $(56.0 \%$ vs. $44.0 \%)$.

\section{Treatment efficacy beyond immunotberapy PD}

The IBP group had a median OS of 26.6 months (95\% CI: 13.3-NA), compared with 9.5 months (95\% CI: 7.7-12.8) in the non-IBP group (HR, 0.40; 95\% CI: 0.23-0.69; $\mathrm{P}<0.001$ ). The 1 -year OS rates were $67 \%$ (95\% CI: $0.53-$ 0.84 ) and 39\% (95\% CI: 0.29-0.52) in the IBP and nonIBP groups, respectively. The median PFS was 8.9 months (95\% CI: 7.4-13.6) in the IBP group and 4.1 months (95\% CI: $3.1-5.2)$ in the non-IBP group (HR, 0.41; $95 \%$ CI: $0.26-0.65 ; \mathrm{P}<0.001)$. The ORR in the IBP group was not statistically different from that in the non-IBP group $(15.4 \%$ vs. $11.6 \%, \mathrm{P}=0.560)$. The $\mathrm{DCR}$ was significantly higher in the IBP group than in the non-IBP group $(89.7 \% \mathrm{vs}$. $61.6 \%, \mathrm{P}<0.001$; Table 2).

By the exploratory subgroup analysis, unweighted for covariates between the IBP and the non-IBP groups, IBP showed a significant benefit in terms of OS and PFS in the overall population and particularly for OS in males, squamous histology, no brain or liver metastases, any age, not beyond $\geq$ the third treatment line, with PR to the previous ICI and monotherapy as previous ICI (Figure S1).

After optimization-based weighting (Table S1), OS was longer for patients in the IBP group than for those in the non-IBP group (mOS: 26.6 vs. 10.7 months; HR, 0.40; 95\% CI: $0.19-0.84 ; \mathrm{P}=0.015)$ and PFS showed similar results (mPFS: 9.7 vs. 4.3 months; HR, 0.28; 95\% CI: 0.15-0.51; $\mathrm{P}<0.001)$. The Kaplan-Meier curves of OS and PFS in both original and weighted data were presented in Figure 1.

\section{Subgroup analysis by the initial immunotherapy regimens}

A subgroup analysis was performed to explore the efficacy of IBP based on patients' initial immunotherapy regimens (monotherapy or combination therapy). Optimizationbased weighting was conducted to balance the distribution of covariates by minimizing the standardized mean difference in the ICI monotherapy and combination therapy subgroups (Tables S2 and S3).

In the ICI monotherapy subgroup, the median OS was statistically significantly different between the IBP and the non-IBP groups (26.6 vs. 10.9 months; HR, 0.31; 95\% CI: $0.11-0.84 ; \mathrm{P}=0.021)$. The median PFS in the IBP group was 10.6 months (95\% CI: 4.8-28.0) compared with 2.1 months (95\% CI: $1.9-4.1$ ) in the non-IBP group (HR, 0.16; 95\% CI: $0.05-0.47 ; \mathrm{P}<0.001$ ).

In the combination therapy subgroup, the IBP had longer OS than the non-IBP patients' group (mOS, $28.8 \mathrm{vs.}$ 11.2 months; HR, 0.42; 95\% CI: 0.18-0.97; $\mathrm{P}=0.042$ ). The median PFS in the IBP group was longer than that in the non-IBP group (mPFS, 8.5 vs. 4.6 months; HR, 0.55; 95\% CI: $0.27-1.16$ ), although the difference was not statistically significant $(\mathrm{P}=0.115)$. The Kaplan-Meier curves of the ICI monotherapy and combination therapy subgroups were shown in Figures 2 and 3.

\section{Subgroup analysis by the best response to initial immunotherapy}

The patients whose best response to initial immunotherapy was $\mathrm{CR} / \mathrm{PR}$ were defined as the response group, while those with $\mathrm{SD} / \mathrm{PD}$ as the non-response group.

For the limited sample size of the response subgroup, the covariate distribution could not be balanced. OS and PFS curves for this subgroup from original unweighted data were shown in Figure S2: the median OS was 28.8 months in IBP compared with 12.4 months in the non-IBP group (HR, 0.08; 95\% CI: 0.01-0.67; P=0.019); the median PFS was 17.0 months in the IBP compared with 2.5 months in the non-IBP group (HR, 0.26; 95\% CI: 0.09-0.79; $\mathrm{P}=0.017)$. 

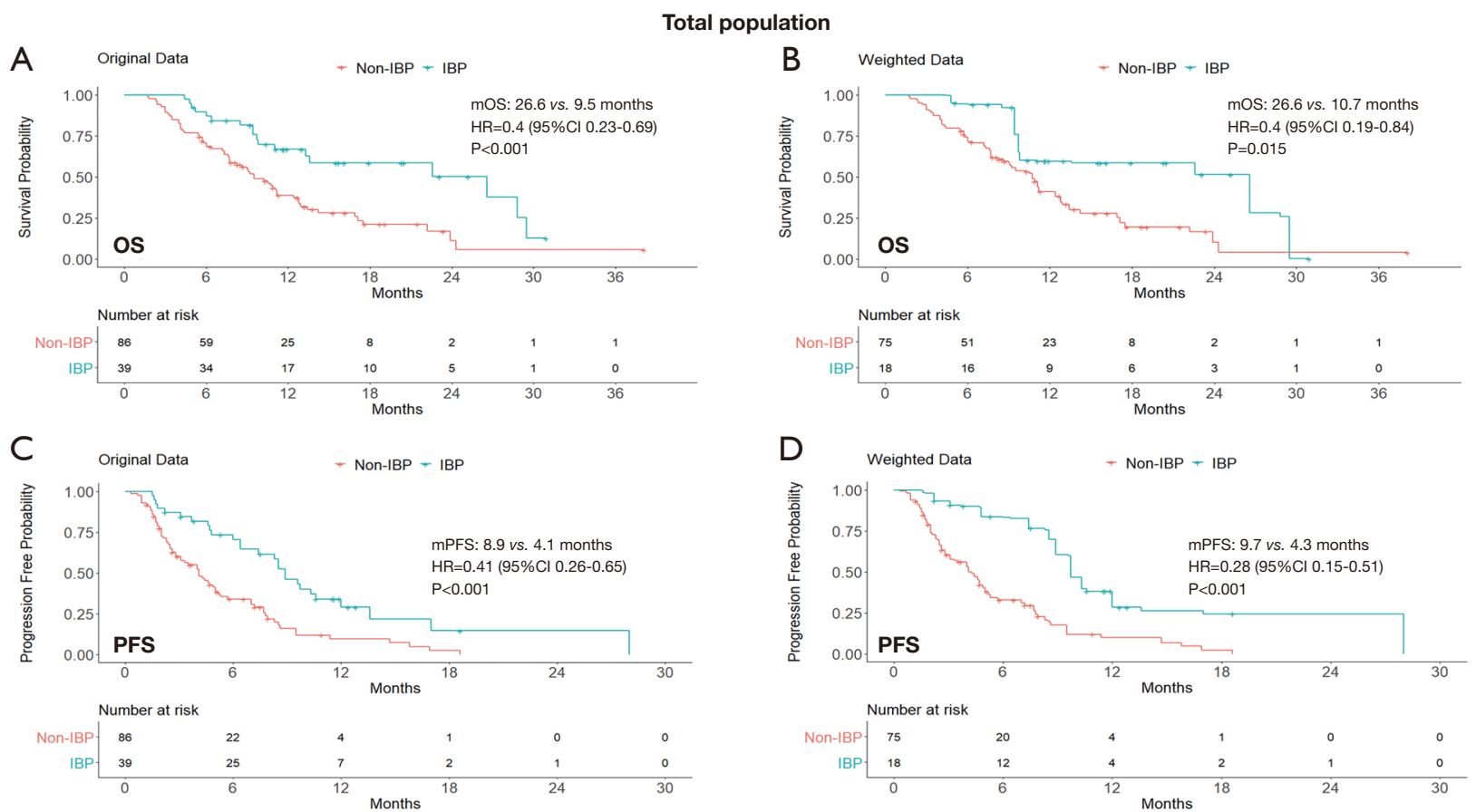

Figure 1 Kaplan-Meier curves of OS (A,B) and PFS (C,D) from original data and weighted data in the total study population. IBP, immunotherapy beyond progression; non-IBP, non-immunotherapy beyond progression; mOS, median overall survival; mPFS, median progression-free survival; HR, hazard ratio; CI, confidence interval.
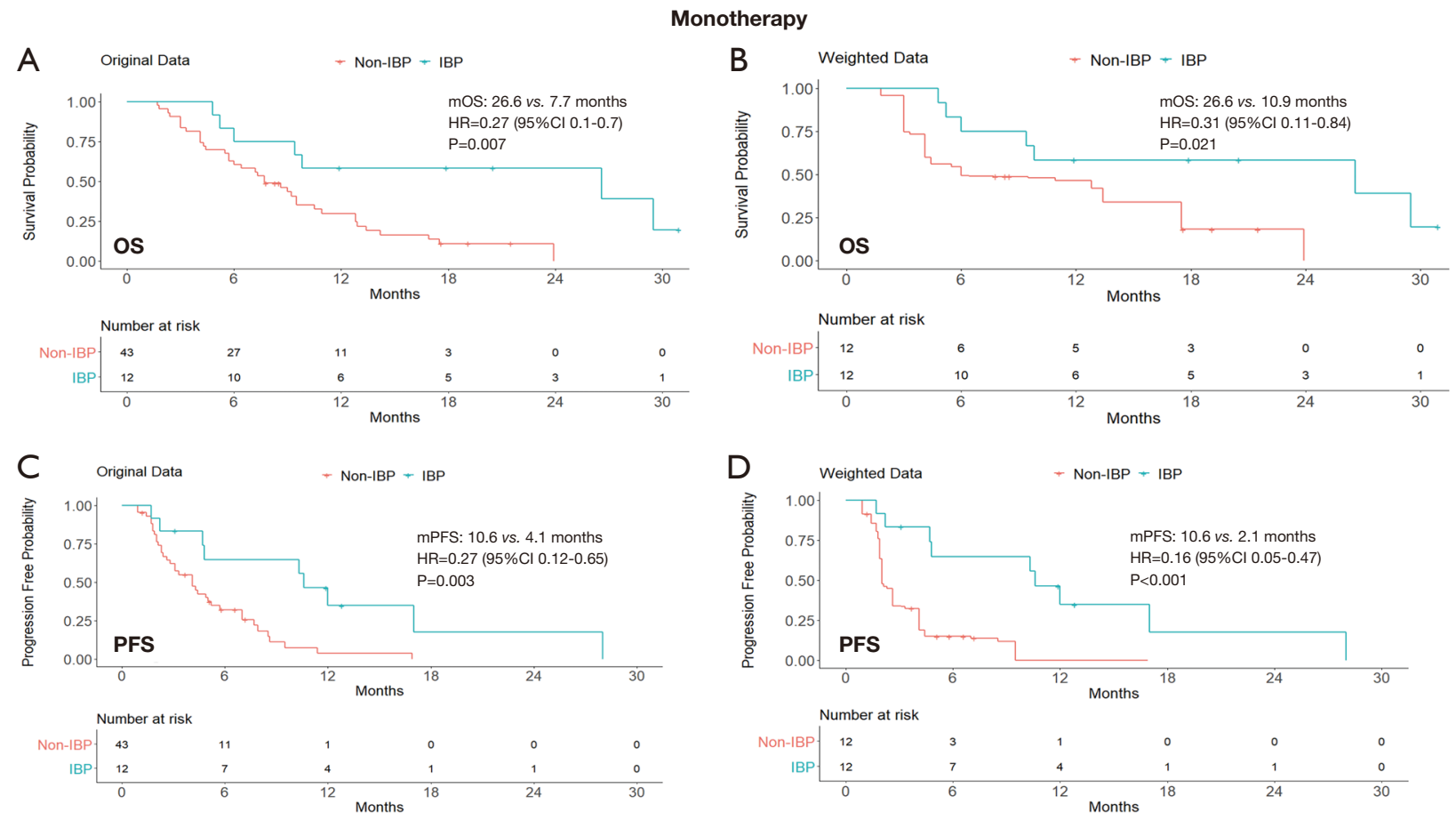

Figure 2 Kaplan-Meier curves of OS (A,B) and PFS (C,D) from original data and weighted data in the initial immune monotherapy subgroup. IBP, immunotherapy beyond progression; non-IBP, non-immunotherapy beyond progression; mOS, median overall survival; mPFS, median progression-free survival; HR, hazard ratio; CI, confidence interval. 

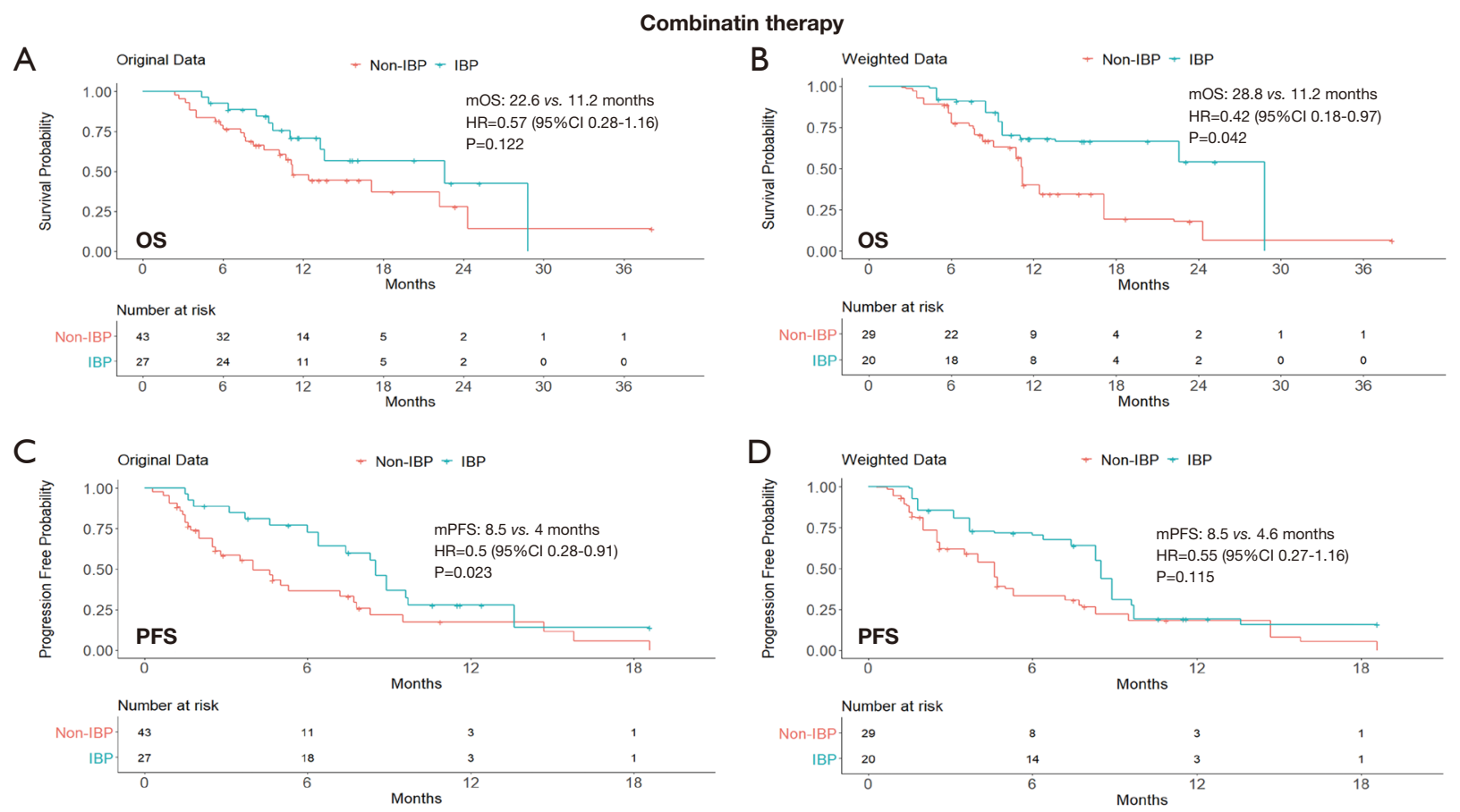

Figure 3 Kaplan-Meier curves of OS (A,B) and PFS (C,D) from original data and weighted data in the initial immune combination therapy subgroup. IBP, immunotherapy beyond progression; non-IBP, non-immunotherapy beyond progression; mOS, median overall survival; mPFS, median progression-free survival; HR, hazard ratio; CI, confidence interval.

After balancing the distribution of covariates in the nonresponse subgroup (Table S4), patients in the IBP group had longer OS (mOS: 26.6 vs. 10.5 months; HR, 0.41; 95\% CI: $0.19-0.87 ; \mathrm{P}=0.020$ ) and PFS (mPFS: 9.7 vs. 4.6 months; HR, 0.35; 95\% CI: $0.19-0.64$; $\mathrm{P}<0.001)$ than those in the non-IBP group (Figure 4).

\section{Discussion}

With the continuous development of immunotherapy for cancer, the treatment choice for patients who experience disease progression to immunotherapy has become a new of unmet need (20). Previous studies have reported that IBP might be effective for patients with aNSCLC (21-25).

In the retrospective OAK study, 322 aNSCLC patients in the atezolizumab group experienced disease progression (26). Among them, patients who continued atezolizumab had a longer OS than those who received other or no further treatments. Similarly, in a retrospective study of more than 4,000 patients with aNSCLC from USA, Stinchcombe et al. (24) reported that IBP patients had a longer OS (11.5 vs. 5.1 months) compared with non-IBP patients. As above mentioned, in a real-world
European series of 60 aNSCLC patients with PD-L1 $\geq 50 \%$ progressed to first-line pembrolizumab, IBP with pembrolizumab and possible local ablative radiotherapies (in $18 / 60$ patients, $30 \%$ ) showed a non-significant difference in post-PD median OS as compared to salvage chemotherapy (in 42/60 patients, $70 \%$ ) (8.1 vs. 6.9 months, $\mathrm{P}=0.08$ ) (17). However, there is little real-world data on IBP for NSCLC patients in China.

In this study, we observed that patients who received IBP showed a survival benefit as compared to those who did not receive this, with longer PFS and OS confirmed after balancing the clinical baseline characteristics between these two groups. To the best of our knowledge, this is the first study using real-world data on IBP for NSCLC in a Chinese population.

According to a subgroup analysis of IBP with nivolumab, Ricciuti et al. (27) reported a survival benefit for patients in the IBP group as compared to non-IBP patients, independently by the best response to the initial immunotherapy, whether disease control or PD. Similarly, our results demonstrated a longer OS and PFS in the IBP than in the non-IBP patients' group, either in the subgroup of patients who responded to the initial immunotherapy or 

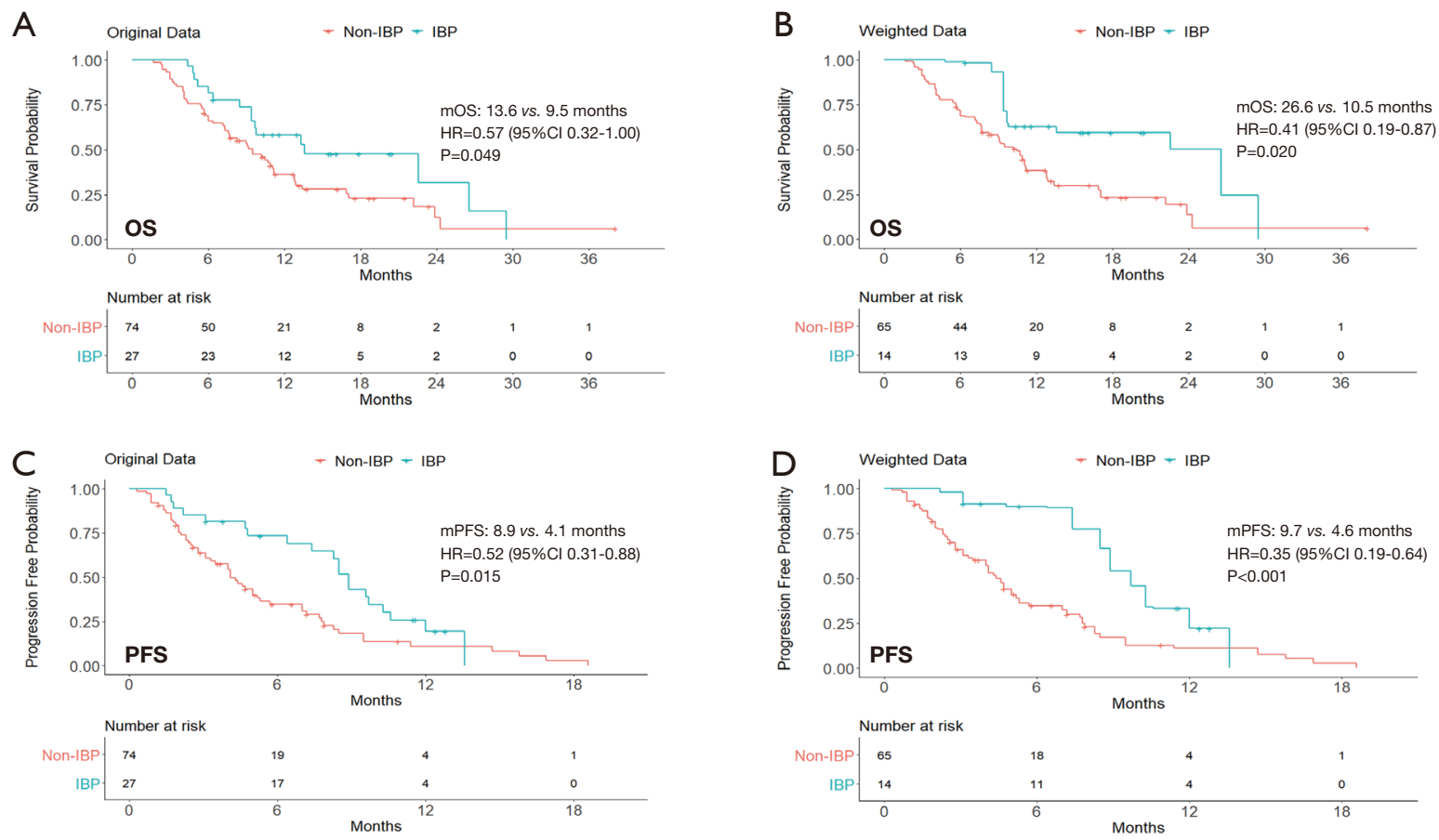

Figure 4 Kaplan-Meier curves of OS (A,B) and PFS (C,D) from original data and weighted data in the subgroup of patients who had SD/ $\mathrm{PD}$ as their best response to previous line of therapy. IBP, immunotherapy beyond progression; non-IBP, non-immunotherapy beyond progression; mOS, median overall survival; mPFS, median progression-free survival; HR, hazard ratio; CI, confidence interval.

in those who showed no response.

Furthermore, we explored whether the initial immunotherapy regimen (monotherapy or combination therapy) affected the outcome of post-progression immunotherapy. By the subgroup analysis, weighted for possible differences in covariates, the benefit from IBP was observed in either the monotherapy or combination ICI therapy subgroups; whilst by the unweighted subgroup analysis, it seemed related to the previous ICI monotherapy only. This possible difference could be explained by the proper weighting of patient characteristics, but the small number of patients in the two subgroups should also be considered. Therefore, the curative effect of immunotherapy remains to be determined and further research on predictive biomarkers is needed.

The mechanism by which some patients who experience progression after initial immunotherapy may benefit from subsequent immunotherapy is still unclear. One reason for this may rely on differences between PD-1/PD-L1 inhibitors (28). And it may take time for the immune system to activate. The interaction between immune system and tumor may lead to fluctuations in clinical efficacy (29). Moreover, to reflect the continuous effect of immunotherapy after the first immunotherapy for PD, and in reference to Ricciuti's study mentioned above (27), we defined whether the duration of immunotherapy after PD exceeded 6 weeks as the boundary between the two groups. Of course, it's not necessarily the best cut-off point in this study. What's more, the current efficacy evaluation criteria for disease response, RECIST v1.1, might not completely applicable to immunotherapy (29). Atypical responses of NSCLC to ICI therapy could, for instance, explain why few patients who achieve a curative effect are mistakenly considered to have disease progression; therefore, the effectiveness of ICIs may be underestimated by RECIST v1.1 (30).

The present study has some limitations. Firstly, although we used an optimization-based method to balance the differences in main baseline clinical characteristics between the groups, other potential factors we might have not considered could have interfered with the conclusions. Secondly, this study was based on the medical records of a single center and the sample size was relatively small; this may undermine the reliability of the results, particularly of those from the subgroup analyses, which should therefore be considered as hypothesis-generating. Thirdly, the retrospective nature of this study has inherent disadvantages 
that may result in recall bias and selection bias. Finally, we cannot adequately estimate the real contribution of salvage chemotherapy following ICIs, although in recent a retrospective series of 342 aNSCLC patients, the reported mOS of 6.8 months and mPFS of 4.1 months seem quite similar to those observed in our non-IBP group (31). Therefore, our findings warrant further validation in multicenter prospective clinical trials with large cohorts.

\section{Conclusions}

IBP may enable patients with aNSCLC to achieve prolonged $\mathrm{OS}$ and PFS. Our findings may suggest new treatment options for patients with aNSCLC who experience disease progression after initial immunotherapy.

\section{Acknowledgments}

We thank Professor Wang for providing assistance and guidance, and the patients and their families for participating in this study. We also appreciate the academic support from AME Lung Cancer Collaborative Group. Funding: None.

\section{Footnote}

Reporting Checklist: The authors have completed the STROBE Reporting Checklist. Available at http://dx.doi. org/10.21037/tlcr-20-1252

Data Sharing Statement: Available at http://dx.doi. org/10.21037/tlcr-20-1252

Conflicts of Interest: All authors have completed the ICMJE uniform disclosure form (available at http://dx.doi. org/10.21037/tlcr-20-1252). PH serves as an unpaid editorial board member of Translational Lung Cancer Research from Feb 2019 to Feb 2021. The other authors have no conflicts of interest to declare.

Ethical Statement: The authors are accountable for all aspects of the work in ensuring that questions related to the accuracy or integrity of any part of the work are appropriately investigated and resolved. The study was conducted in accordance with the Declaration of Helsinki (as revised in 2013). The study was approved by the Ethics Committee of PLA General Hospital (S2018-141-01) and individual consent for this retrospective analysis was waived.
Open Access Statement: This is an Open Access article distributed in accordance with the Creative Commons Attribution-NonCommercial-NoDerivs 4.0 International License (CC BY-NC-ND 4.0), which permits the noncommercial replication and distribution of the article with the strict proviso that no changes or edits are made and the original work is properly cited (including links to both the formal publication through the relevant DOI and the license). See: https://creativecommons.org/licenses/by-nc-nd/4.0/.

\section{References}

1. Bray F, Ferlay J, Soerjomataram I, et al. Global cancer statistics 2018: GLOBOCAN estimates of incidence and mortality worldwide for 36 cancers in 185 countries. CA Cancer J Clin 2018;68:394-424.

2. Rosell R, Karachaliou N. Large-scale screening for somatic mutations in lung cancer. Lancet 2016;387:1354-6.

3. Garon EB, Rizvi NA, Hui R, et al. Pembrolizumab for the treatment of non-small-cell lung cancer. N Engl J Med 2015;372:2018-28.

4. Cummings AL, Garon EB. KEYNOTE-042 rolls back programmed cell death ligand 1 threshold for non-small cell lung cancer pembrolizumab monotherapy without new insight into those deriving benefit. Transl Lung Cancer Res 2019;8:S403-6.

5. Brahmer J, Reckamp KL, Baas P, et al. Nivolumab versus Docetaxel in Advanced Squamous-Cell Non-Small-Cell Lung Cancer. N Engl J Med 2015;373:123-35.

6. Areses Manrique MC, Mosquera Martínez J, García González J, et al. Real world data of nivolumab for previously treated non-small cell lung cancer patients: a Galician lung cancer group clinical experience. Transl Lung Cancer Res 2018;7:404-15.

7. Reck M, Rodriguez-Abreu D, Robinson AG, et al. Pembrolizumab versus Chemotherapy for PD-L1Positive Non-Small-Cell Lung Cancer. N Engl J Med 2016;375:1823-33.

8. Mok TSK, Wu YL, Kudaba I, et al. Pembrolizumab versus chemotherapy for previously untreated, PD-L1expressing, locally advanced or metastatic non-small-cell lung cancer (KEYNOTE-042): a randomised, open-label, controlled, phase 3 trial. Lancet 2019;393:1819-30.

9. Rittmeyer A, Barlesi F, Waterkamp D, et al. Atezolizumab versus docetaxel in patients with previously treated non-small-cell lung cancer (OAK): a phase 3, openlabel, multicentre randomised controlled trial. Lancet 2017;389:255-65. 
10. Ribas A, Wolchok JD. Cancer immunotherapy using checkpoint blockade. Science 2018;359:1350-5.

11. Beaver JA, Hazarika M, Mulkey F, et al. Patients with melanoma treated with an anti-PD-1 antibody beyond RECIST progression: a US Food and Drug Administration pooled analysis. Lancet Oncol 2018;19:229-39.

12. George S, Motzer RJ, Hammers HJ, et al. Safety and Efficacy of Nivolumab in Patients With Metastatic Renal Cell Carcinoma Treated Beyond Progression: A Subgroup Analysis of a Randomized Clinical Trial. JAMA Oncol 2016;2:1179-86.

13. Rebuzzi SE, Bregni G, Grassi M, et al. Immunotherapy beyond progression in advanced renal cell carcinoma: a case report and review of the literature. Immunotherapy 2018;10:1123-32.

14. Escudier B, Motzer RJ, Sharma P, et al. Treatment Beyond Progression in Patients with Advanced Renal Cell Carcinoma Treated with Nivolumab in CheckMate 025. Eur Urol 2017;72:368-76.

15. Long GV, Weber JS, Larkin J, et al. Nivolumab for Patients With Advanced Melanoma Treated Beyond Progression: Analysis of 2 Phase 3 Clinical Trials. JAMA Oncol 2017;3:1511-9.

16. Herbst RS, Garon EB, Kim DW, et al. Long-Term Outcomes and Retreatment Among Patients With Previously Treated, Programmed Death-Ligand 1Positive, Advanced Non-Small-Cell Lung Cancer in the KEYNOTE-010 Study. J Clin Oncol 2020;38:1580-90.

17. Metro G, Addeo A, Signorelli D, et al. Outcomes from salvage chemotherapy or pembrolizumab beyond progression with or without local ablative therapies for advanced non-small cell lung cancers with PD-L1 $\geq 50 \%$ who progress on first-line immunotherapy: real-world data from a European cohort. J Thorac Dis 2019;11:4972-81.

18. Eisenhauer EA, Therasse P, Bogaerts J, et al. New response evaluation criteria in solid tumours: revised RECIST guideline (version 1.1). Eur J Cancer 2009;45:228-47.

19. Zubizarreta JR. Stable Weights that Balance Covariates for Estimation With Incomplete Outcome Data. Journal of the American Statistical Association 2015;110:910-22.

20. Blumenthal GM, Theoret MR, Pazdur R. Treatment Beyond Progression With Immune Checkpoint InhibitorsKnown Unknowns. JAMA Oncol 2017;3:1473-4.

21. Fujita K, Uchida N, Yamamoto Y, et al. Retreatment With Anti-PD-L1 Antibody in Advanced Non-small Cell Lung Cancer Previously Treated With Anti-PD-1 Antibodies. Anticancer Res 2019;39:3917-21.

22. Fujita K, Uchida N, Kanai O, et al. Retreatment with pembrolizumab in advanced non-small cell lung cancer patients previously treated with nivolumab: emerging reports of 12 cases. Cancer Chemother Pharmacol 2018;81:1105-9.

23. Niki M, Nakaya A, Kurata T, et al. Immune checkpoint inhibitor re-challenge in patients with advanced non-small cell lung cancer. Oncotarget 2018;9:32298-304.

24. Stinchcombe TE, Miksad RA, Gossai A, et al. RealWorld Outcomes for Advanced Non-Small Cell Lung Cancer Patients Treated With a PD-L1 Inhibitor Beyond Progression. Clin Lung Cancer 2020;21:389-394.e3.

25. Haddad R, Concha-Benavente F, Blumenschein G, Jr., et al. Nivolumab treatment beyond RECIST-defined progression in recurrent or metastatic squamous cell carcinoma of the head and neck in CheckMate 141: A subgroup analysis of a randomized phase 3 clinical trial. Cancer 2019;125:3208-18.

26. Gandara DR, von Pawel J, Mazieres J, et al. Atezolizumab Treatment Beyond Progression in Advanced NSCLC: Results From the Randomized, Phase III OAK Study. J Thorac Oncol 2018;13:1906-18.

27. Ricciuti B, Genova C, Bassanelli M, et al. Safety and Efficacy of Nivolumab in Patients With Advanced Nonsmall-cell Lung Cancer Treated Beyond Progression. Clin Lung Cancer 2019;20:178-185.e2.

28. Banna GL, Cantale O, Bersanelli M, et al. Are anti-PD1 and anti-PD-L1 alike? The non-small-cell lung cancer paradigm. Oncol Rev 2020;14:490.

29. Queirolo P, Spagnolo F. Atypical responses in patients with advanced melanoma, lung cancer, renal-cell carcinoma and other solid tumors treated with anti-PD-1 drugs: A systematic review. Cancer Treat Rev 2017;59:71-8.

30. Kim HK, Heo MH, Lee HS, et al. Comparison of RECIST to immune-related response criteria in patients with non-small cell lung cancer treated with immunecheckpoint inhibitors. Cancer Chemother Pharmacol 2017;80:591-8.

31. Bersanelli M, Buti S, Giannarelli D, et al. Chemotherapy in non-small cell lung cancer patients after prior immunotherapy: The multicenter retrospective CLARITY study. Lung Cancer 2020;150:123-31.

Cite this article as: Ge $\mathrm{X}$, Zhang Z, Zhang S, Yuan F, Zhang F, Yan X, Han X, Ma J, Wang L, Tao H, Li X, Zhi X, Huang Z, Hofman P, Prelaj A, Banna GL, Mutti L, Hu Y, Wang J. Immunotherapy beyond progression in patients with advanced non-small cell lung cancer. Transl Lung Cancer Res 2020;9(6):2391-2400. doi: 10.21037/tlcr-20-1252 


\section{Supplementary}

Table S1 Summary of baseline characteristics of all patients

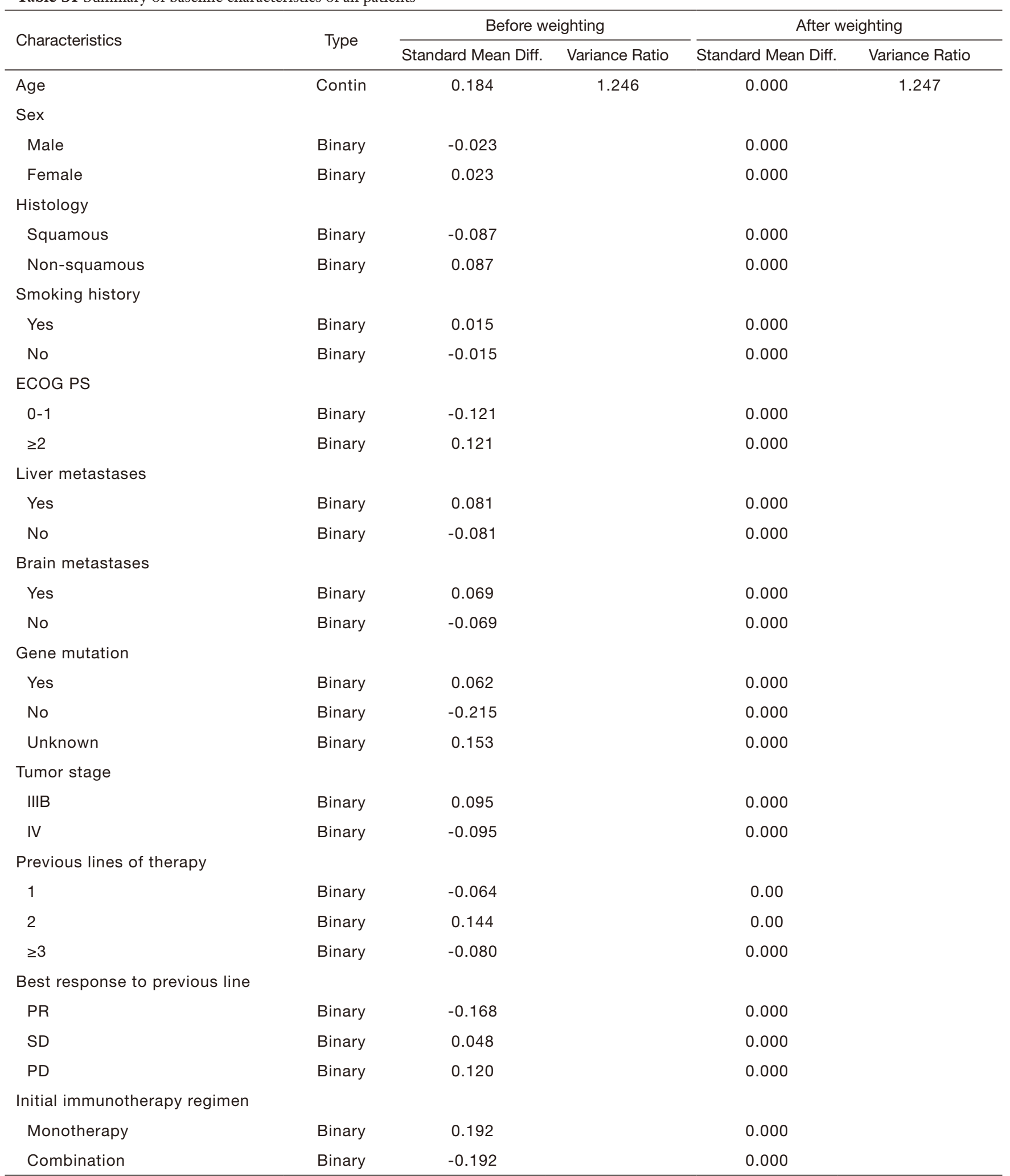

Contin: continuous variable; Binary: binary variable; ECOG PS, Eastern Cooperative Oncology Group Performance Status; PR, partial response; $\mathrm{SD}$, stable disease; $\mathrm{PD}$, progressive disease. 
Table S2 Summary of the baseline characteristics of patients in the initial immune monotherapy subgroup

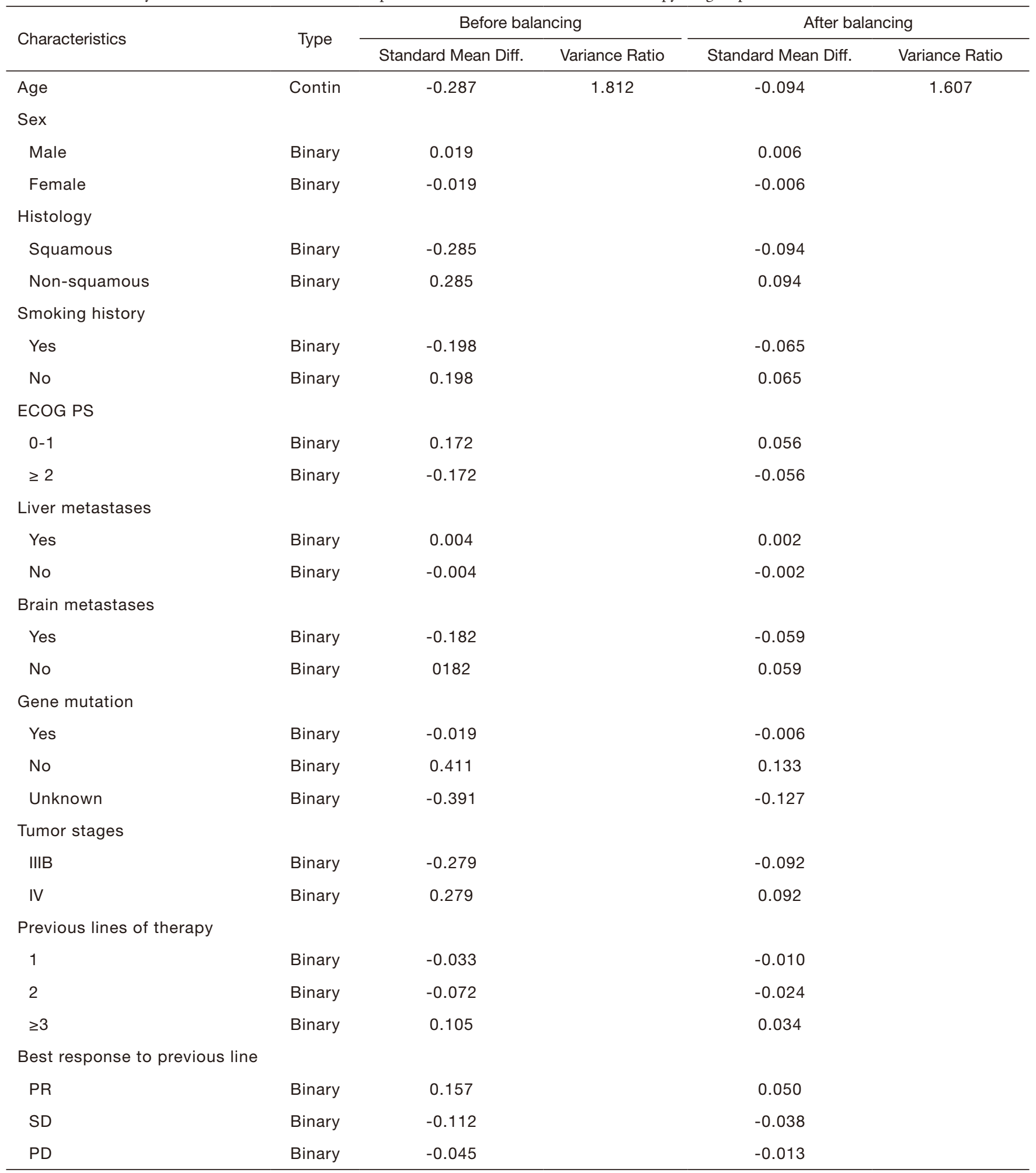

Contin: continuous variable; Binary: binary variable; ECOG PS, Eastern Cooperative Oncology Group Performance Status; PR, partial response; $\mathrm{SD}$, stable disease; $\mathrm{PD}$, progressive disease. 
Table S3 Summary of the baseline characteristics of patients in the initial immune combination therapy subgroup

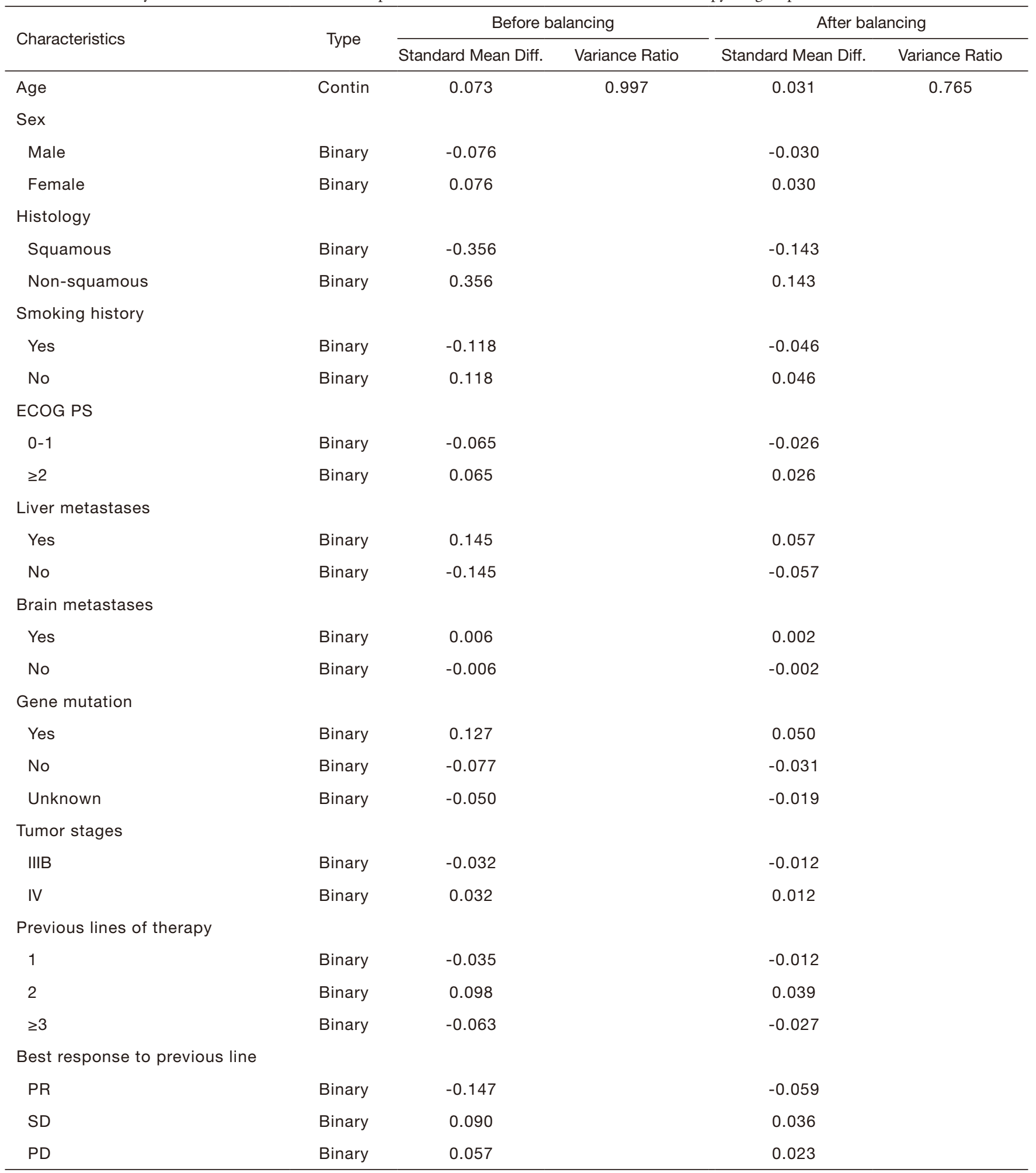

Contin: continuous variable; Binary: binary variable; ECOG PS, Eastern Cooperative Oncology Group Performance Status; PR, partial response; $\mathrm{SD}$, stable disease; $\mathrm{PD}$, progressive disease. 
Table S4 Summary of the baseline characteristics of patients who had non-PR (SD/PD) as their best response to previous line

\begin{tabular}{|c|c|c|c|c|c|}
\hline Characteristics & Type & \multicolumn{2}{|c|}{ Before balancing } & \multicolumn{2}{|c|}{ After balancing } \\
\hline Age & Contin & 0.148 & 1.569 & 0.032 & 1.409 \\
\hline \multicolumn{6}{|l|}{ Sex } \\
\hline Male & Binary & -0.048 & & -0.009 & \\
\hline \multicolumn{6}{|l|}{ Histology } \\
\hline Squamous & Binary & -0.029 & & -0.006 & \\
\hline Non-squamous & Binary & 0.029 & & 0.006 & \\
\hline \multicolumn{6}{|l|}{ Smoking history } \\
\hline \multicolumn{6}{|l|}{ ECOG PS } \\
\hline $0-1$ & Binary & -0.115 & & -0.024 & \\
\hline$\geq 2$ & Binary & 0.115 & & 0.024 & \\
\hline \multicolumn{6}{|l|}{ Liver metastases } \\
\hline Yes & Binary & 0.092 & & 0.018 & \\
\hline No & Binary & -0.092 & & -0.018 & \\
\hline \multicolumn{6}{|l|}{ Brain metastases } \\
\hline Yes & Binary & -0.023 & & -0.005 & \\
\hline \multicolumn{6}{|l|}{ Gene mutation } \\
\hline \multicolumn{6}{|l|}{ Tumor stages } \\
\hline IIIB & Binary & 0.156 & & 0.032 & \\
\hline IV & Binary & -0.156 & & -0.032 & \\
\hline \multicolumn{6}{|c|}{ Previous lines of therapy } \\
\hline 1 & Binary & 0.058 & & 0.013 & \\
\hline 2 & Binary & 0.193 & & 0.040 & \\
\hline$\geq 3$ & Binary & -0.251 & & -0.053 & \\
\hline \multicolumn{6}{|c|}{ Initial immunotherapy regimen } \\
\hline Monotherapy & Binary & 0.194 & & 0.040 & \\
\hline Combination & Binary & -0.194 & & -0.040 & \\
\hline
\end{tabular}

Contin: continuous variable; Binary: binary variable; ECOG PS, Eastern Cooperative Oncology Group Performance Status; PR, partial response; $\mathrm{SD}$, stable disease; $\mathrm{PD}$, progressive disease. 
A

Overall
Sex
Male
Female
Age
$<65$
$\geq 65$
Smoking
Never Smoke
Smoke
Histology
Non-squamous
Squamous
Brian Metastases
No
Yes
Liver Metastases
No
Yes
Initial IO line
1 line
2 line
$\geq 3$ lines
Initial IO response
PR
SD
PD
Initial IO Type
Mono
Combo

\begin{tabular}{cc} 
Total & non-IBP \\
125 & 86 \\
& \\
91 & 62 \\
34 & 24 \\
& \\
94 & 63 \\
31 & 23 \\
50 & 34 \\
75 & 52 \\
78 & 56 \\
47 & 30 \\
& \\
87 & 58 \\
38 & 28 \\
102 & 68 \\
23 & 18 \\
33 & 21 \\
38 & 30 \\
54 & 35 \\
24 & 12 \\
49 & 35 \\
52 & 39 \\
55 & 43 \\
70 & 43 \\
\hline &
\end{tabular}

IBP
39
29

HR
0.4

0.4

0.37

0.47

$16 \quad 0.29$

23

22
17

$29 \quad 0.35$

10

$34 \quad 0.38$

5

$\begin{array}{cc}12 & \\ 8 & 0 \\ 19 & 0.2\end{array}$

$19 \quad 0.2$

$\begin{array}{ll}12 & 0.08 \\ 14 & 0.82\end{array}$

$\begin{array}{ll}14 & 0.82 \\ 13 & 0.82\end{array}$

$\begin{array}{ll}12 & 0.27 \\ 27 & 0.57\end{array}$ $\begin{array}{cc}31 & 0.47 \\ 8 & 0.12\end{array}$
$95 \% \mathrm{Cl}$

(0.12,1.07)

$(0.27,0.83)$

$(0.02,0.94)$

$(0.12,0.72)$

$(0.2,0.89)$

(0.27,1.01)

$(0.1,0.63)$

$(0.17,0.69)$

$(0.29,1.61)$

$(0.21,0.68)$

$(0.25,3.12)$

$(0$, Inf)

$(0.07,0.6)$

$(0.07,0.6)$

$(0.01,0.67)$

(0.36,1.87)

(0.36,1.87)

$(0.1,0.7)$
(0.23,0.69)

$(0.22,0.8)$

$(0.28,1.16)$

B

Overall
Sex
Male
Female
Age
$<65$
$\geq 65$
Smoking
Never Smoke
Smoke
Histology
Non-squamous
Squamous
Brian Metastases
No
Yes
Liver Metastases
No
Yes
Initial IO line
1 line
2 line
$\geq 3$ lines
Initial IO response
PR
SD
PD
Initial IO Type
Mono
Combo

$\begin{array}{cccc}\text { Total } & \text { non-IBP } & \text { IBP } & \text { HR } \\ 125 & 86 & 39 & 0.41 \\ & & & \\ 91 & 62 & 29 & 0.47 \\ 34 & 24 & 10 & 0.28 \\ & & & \\ 94 & 63 & 31 & 0.46 \\ 31 & 23 & 8 & 0.13 \\ & & & \\ 50 & 34 & 16 & 0.32 \\ 75 & 52 & 23 & 0.53 \\ 78 & 56 & 22 & 0.38 \\ 47 & 30 & 17 & 0.49 \\ & & & \\ 87 & 58 & 29 & 0.43 \\ 38 & 28 & 10 & 0.4 \\ & & & \\ 102 & 68 & 34 & 0.37 \\ 23 & 18 & 5 & 1.32 \\ 33 & 21 & 12 & 0.55 \\ 38 & 30 & 8 & 0.29 \\ 54 & 35 & 19 & 0.29 \\ 24 & 12 & 12 & 0.26 \\ 49 & 35 & 14 & 0.51 \\ 52 & 39 & 13 & 0.51 \\ 55 & 43 & 12 & 0.27 \\ 70 & 43 & 27 & 0.5\end{array}$

$95 \% \mathrm{Cl}$
$(0.26,0.65)$

$(0.28,0.8)$

$(0.1,0.76)$

$(0.28,0.77)$

(0.03,0.62)

$(0.16,0.67)$

$(0.29,0.97)$

$(0.21,0.7)$

$(0.24,1.03)$

$(0.25,0.75)$

(0.17,0.94)

$(0.22,0.62)$

$(0.41,4.24)$

$(0.2,1.53)$

$(0.11,0.73)$

$(0.11,0.73)$

$(0.09,0.79)$

$(0.24,1.07)$

(0.24,1.07)

$(0.12,0.65)$

$(0.28,0.91)$

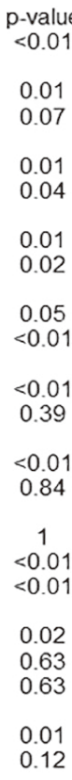

P-value
$<0.01$

0.01
0.07

0.01

0.04

0.02

0.05

$<0.01$

0.39

$<0.01$

0.84

1

$<0.01$

0.02

0.63
0.63

0.01
0.12
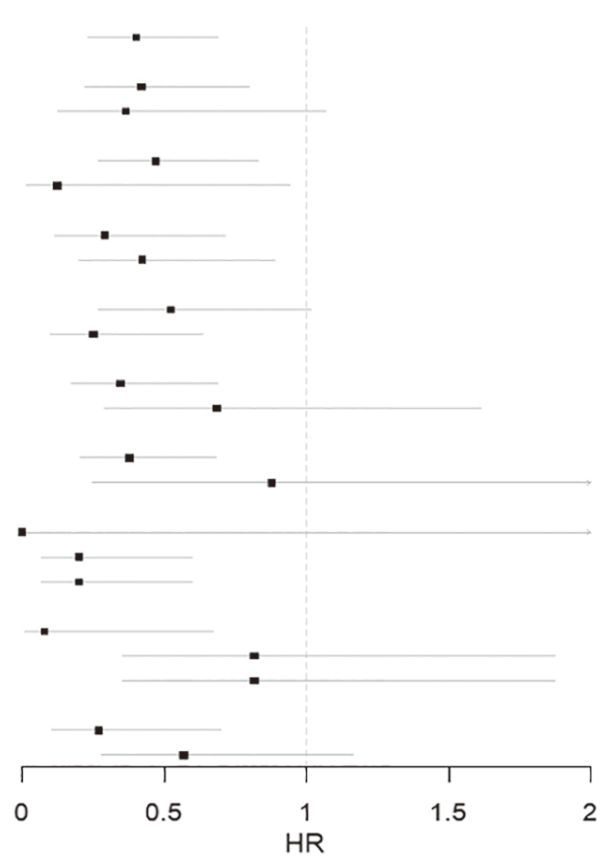

p-value

$<0.01$

0.01

0.01

$<0.01$

0.01

$<0.01$

0.04

$<0.01$
0.06

$<0.01$

$<0.01$
0.03

$<0.01$
0.64

0.25

0.25

0.01
0.01

0.02

0.08

$<0.01$
0.02

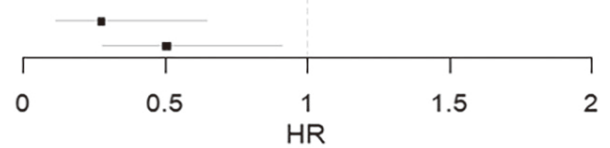

Figure S1 Forest plots of OS (A) and PFS (B) in the IBP and non-IBP groups. 
A

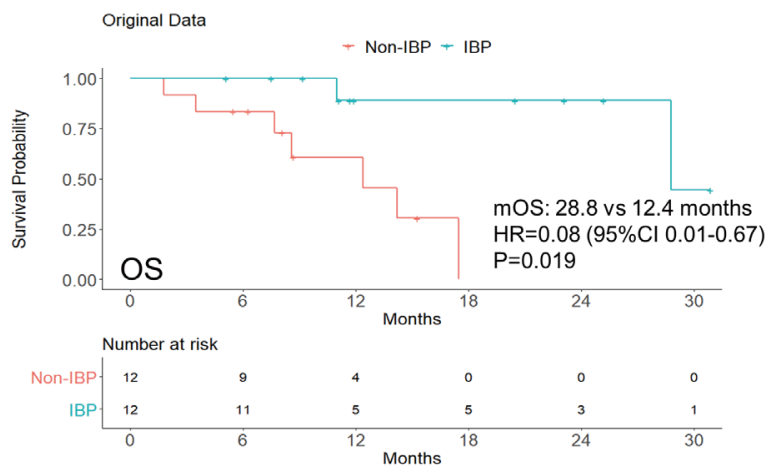

B

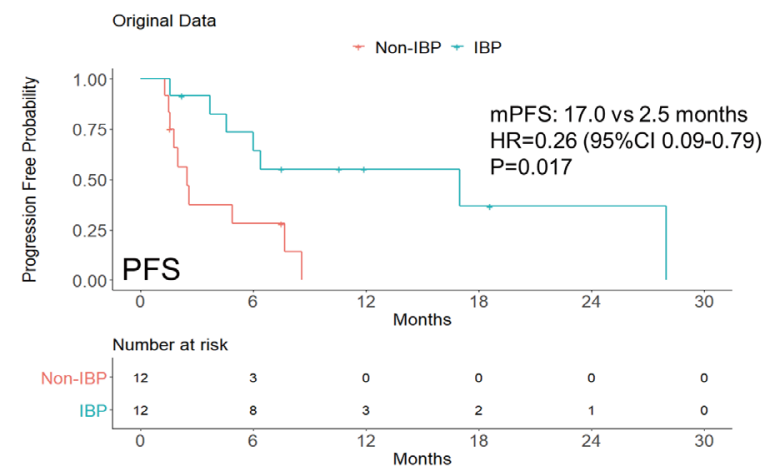

Figure S2 Kaplan-Meier curves of OS (A) and PFS (B) from original data in the subgroup of patients who had PR as their best response to previous line. 